\title{
Retrospective Epidemiological Investigation on Nosocomial Neonatal Sepsis in Shaanxi Province (2008-2010)
}

\section{Li Zhang ${ }^{1 *}$, Qin Zhang2\#, Heqin Li3", Wenjing Wang4\#, Wenping Song5\#, Huirong Li6", Xun Jiang"\#, Liming $\mathrm{Ni}^{8 \#}, \mathrm{Li} \mathrm{Liu}^{9}$, Yaping Wang10\#}

${ }^{1}$ Department of Neonatology, Mother and Children's Healthcare Hospital of Shaanxi Province, Xi'an, China

${ }^{2}$ Department of Neonatology, People's Hospital of Shaanxi Province, Xi'an, China

${ }^{3}$ Department of Neonatology, Mother and Children Healthcare Hospital of Baoji City, Baoji, China

${ }^{4}$ Department of Neonatology, Xi'an Children's Hospital, Xi'an, China

${ }^{5}$ Department of Neonatology, Mother and Children Healthcare Hospital of Weinan City, Weinan, China

${ }^{6}$ Department of Neonatology, Yulin Children's Hospital, Yulin, China

${ }^{7}$ Department of Neonatology, Tangdu Hospital, Fourth Military Medical University, Xi'an, China

${ }^{8}$ Department of Neonatology, Fourth Hospital of Xi'an City, Xi'an, China

${ }^{9}$ Department of Neonatology, First Affiliated Hospital of Xi'an Jiao Tong University, Xi'an, China

${ }^{10}$ Department of Neonatology, Xianyang First Hospital, Xianyang, China

Email:*sshr_lily@163.com

How to cite this paper: Zhang, L., Zhang, Q., Li, H.Q., Wang, W.J., Song, W.P., Li, H.R., Jiang, X., Ni, L.M., Liu, L. and Wang, Y.P. (2016) Retrospective Epidemiological Investigation on Nosocomial Neonatal Sepsis in Shaanxi Province (2008-2010). Open Journal of Pediatrics, 6, 262-273. http://dx.doi.org/10.4236/ojped.2016.64037

Received: September 25, 2016

Accepted: October 31, 2016

Published: November 3, 2016

Copyright $\odot 2016$ by authors and Scientific Research Publishing Inc. This work is licensed under the Creative Commons Attribution International License (CC BY 4.0).

http://creativecommons.org/licenses/by/4.0/ (c) (i) Open Access

\section{Abstract}

Objective: This study assessed the incidence, mortality, and pathogens associated with nosocomial neonatal sepsis (NNS) in Shaanxi Province to improve the prevention and control of NNS. Methods: Nine large neonatal departments in Shaanxi Province participated in this retrospective epidemiological investigation of NNS during the period of 2008-2010, using standardized protocol. The incidence, mortality, pathogens, antibiotic sensitivity, clinical characteristics, prognosis, and costs were analyzed. Results: Of 16,642 neonates admitted to neonatal departments in the 9 hospitals during 2008-2010, there were 139 cases of NNS, with incidence of $8.3 \%$ and mortality of $20.8 \%$. The predominant pathogens were Gram-negative bacteria, accounting for $67.6 \%$ of positive cultures. Gram-positive bacteria accounted for $24.5 \%$ and fungal infection accounted for $7.9 \%$. The most common Gram-negative species were Klebsiella pneumonia (22.3\%), Escherichia coli (15.1\%), and Enterobacter cloacae (8.6\%). The dominant Gram-positive species identified were coagulase negative staphylococcal species (CONS) (8.6\%), followed by Staphylococcus aureus (7.9\%). The recovered bacterial pathogens demonstrated varying antibiotic resis-

\footnotetext{
"All authors equally contributed to the study and belong to Shaanxi Collaborative Study Group for Nosocomial Neonatal Sepsis.
} 
tance, but no meropenem-or-vancomycin-resistant strains were detected. Preterm and full-term infants showed significant difference in clinical manifestations, laboratory findings, mortality, and cost. Conclusion: NNS in the hospitals of Shaanxi Province showed a decreasing trend from 2008 to 2010, but the mortality did not reduce significantly. The predominant bacteria were Gram negative, and pathogens were found to have varying antibiotic resistance. The preterm group had higher mortality and costs than the full-term infants. Therefore, effective measures should be taken to control NNS, especially in preterm infants.

\section{Keywords}

Incidence, Mortality, Pathogens, Nosocomial, Infection, Neonatal Sepsis

\section{Introduction}

About four million neonatal deaths occur worldwide annually, of which 900,000 are caused by sepsis and 450 newborns die every hour mainly from preventable causes [1]. Three major causes of neonatal deaths, including infections, complications of preterm birth, and intrapartum-related neonatal deaths, account for almost $90 \%$ of all neonatal deaths [2]. Almost all (99\%) neonatal deaths occur in developing countries, and China is among the top 10 countries that contribute to the largest numbers of neonatal deaths [3]. Without addressing these concerns, the Millennium Development Goal for Child Survival cannot be achieved. Developed countries have a better grasp of epidemiological information. Although China has a large number of newborn babies, the research data in this field is very limited [4]. In October 2008, there were 8 neonatal deaths because of hospital-acquired infection in a tertiary hospital in Shaanxi Province, which triggered Chinese Ministry of Health to improve controlling of hospital-acquired infection among neonates by strengthening administrative management. To collect the clinical data of from 2008 to 2010, this study assessed the incidence, mortality, and pathogens associated with nosocomial neonatal sepsis (NNS) in Shaanxi Province to improve the prevention and control of NNS.

\section{Methods}

\subsection{Study Design and Data Collection}

The present research, conducted by the Neonatal Network Organization in Shaanxi Province, was a retrospective epidemiological survey that analyzed the overall data of NNS in 9 participant hospitals from Jan. 1, 2008, to Dec. 31, 2010. These 9 hospitals included 2 children's hospitals, 3 maternal and child health hospitals, and 5 general hospitals.

During the research period, the recruited patients' medical records were filled out by the neonatologists, Including perinatal risk factors, gestational age, birth weight, age of 
onset, clinical manifestation, laboratory examination, prognosis and disease burden, pathogen, antibiotic susceptibility test results. Bacterial culture was prepared using an automatic bacteria culture instrument and antibiotic sensitivity testing was performed by disc diffusion method [5]. The research was approved by the ethics committee of the Mother and Children's Healthcare Hospital of Shaanxi Province.

\subsection{Definition}

NNS is defined as follows, hospitalized neonates with clinical signs of sepsis and a positive culture were included in the study in the event of any of the following: sepsis-acquired infections during parturition and postpartum, which occurred $48 \mathrm{~h}$ after birth; sepsis related with last hospitalization; or new pathogens different from original pathogen infection (excluding pollution and original mixed infection). Cases of neonatal trans-placental obtained sepsis were excluded (onset within $48 \mathrm{~h}$ after birth [6] [7].

\subsection{Statistical Analysis}

The SPSS 17.0 Statistical Software Package (SPSS Inc, Chicago, IL) was used for data analysis. Continuous variables were summarized as median with inter-quartile range (IQR) or range and mean with SD. Categorical data were presented as absolute numbers and proportions. Comparisons of the categorical variables were performed with the Chi square test or Fisher's exact test.

\section{Results}

\subsection{General Information}

A total of 16,642 admitted newborns were surveyed, among which 139 were cultureproven NNS cases, including 97 male and 42 female infants, 84 preterm and 55 full-term, with gestational age of 27 to 42 weeks (Figure 1). Only $40 \%$ of the cases enjoyed the new type of rural cooperative medical care (NCMS) support which cover $30 \%-50 \%$ of medical cost for family.

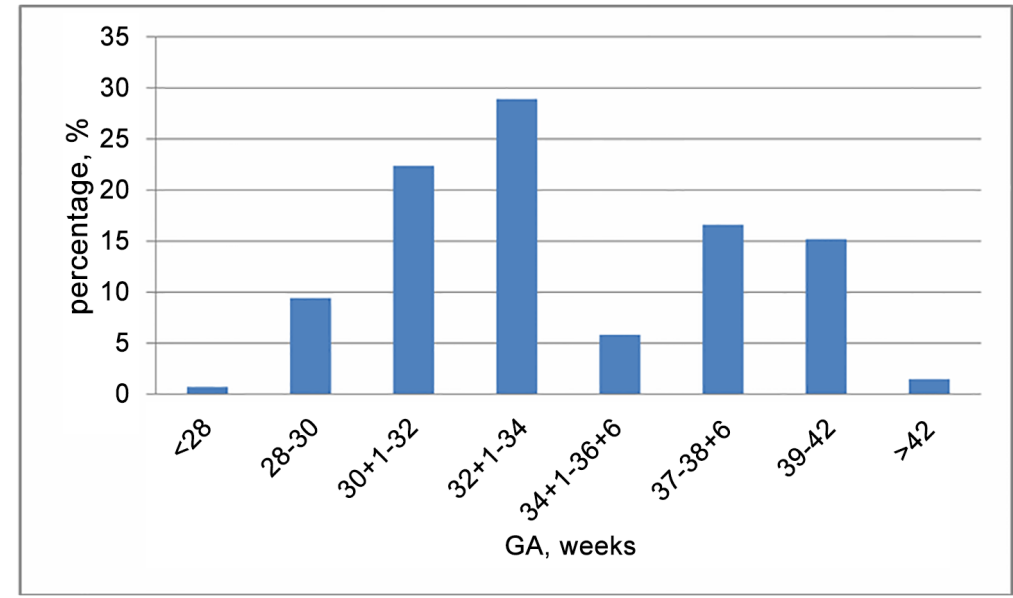

Figure 1. Gestational age distribution of NNS in Shaanxi Province: 2008-2010. 
Among the 93 (low birth weight (LBW) infants, 2\% (2/93) were extremly low birth weight (ELBW) and 27.9\% (26/93) were very low birth weight (VLBW) In all NNS cases, the cesarean section rate was $61.8 \%$ (86/139), NICU treatment with incubators was performed in $71.9 \%$ (100/139), respiratory support including ventilation and oxygen therapy was provided in $20.1 \%$ (28/139), birth asphyxia developed in $8.6 \%(12 / 139)$, premature rupture of membranes was reported in $14.4 \%$ (20/139), and amniotic fluid contamination was reported in 10\% (14/139). After admission, 88.4\% (123/139) infants received antibiotic treatment, $50.4 \%$ (62/139) of whom were given prophylactic antibiotics. Third-generation cephalosporin or enzyme penicillin was used for therapeutic or prophylactic treatment in $69.1 \%(85 / 123)$ of infants, ampicillin in $9.8 \%(12 / 123)$ of infants, and none of them were treated with gentamicin. Up to $49.6 \%(69 / 139)$ received two antibiotic therapies. As many as $93.5 \%$ (130/139) of the infants were formula fed, and only $1.4 \%(2 / 139)$ were exclusively breast fed. The average time of starting enteral nutrition feeding was 2.5 days and the total enteral nutrition time was 19.7 days. Up to 88.4\% (123/139) received intravenous nutrition therapies, 23\% (32/139) used PICC, and $18.7 \%(6 / 32)$ developed catheter-related sepsis. Meanwhile, one nursing staff took care of $6-8$ infants during daytime or 8-12 infants at night in these hospitals.

\subsection{Incidence and Mortality}

We identified 139 cases of culture-proven NNS among admitted neonates in the 9 hospitals during 2008-2010 (Table 1). The overall incidence of NNS was $0.83 \%$ and the mortality was $20.8 \%$ during the period. With comprehensive infection control measures, incidence and mortality of NNS decreased gradually but there were not statistically significant difference respectively in Shaanxi Province $(P=0.081, P=0.817)$. The overall incidence of hospital infection among the admitted newborns to the total admissions in the neonatology departments was 38.9\%. Although the proportion of NNS increased among overall hospital infections after strengthened prevention and control, there was no statistically significant difference $(P=0.618)$.

\subsection{Clinical Manifestations, Laboratory Examinations and Outcomes}

Despite atypical symptoms in NNS, the onset in full-term and preterm infants did not show the same initial clinical manifestations: Apnea, poor feeding, poor response, weight loss, and abdominal distension occurred in the preterm group, while the fullterm group initially showed poor feeding, jaundice exacerbation, weight loss, and poor response. There was no significant difference between the two groups in terms of poor feeding, dyspnea stressed, no weight increase, or abnormal defecation. The mortality rate was high, abandoning treatment occurred quite frequently, and the mortality of the preterm infants was much higher than that of the full-term infants, resulting in a heavier disease burden (Table 2).

Laboratory examinations also showed different characteristics (Table 3). To some extent, the laboratory tests in NNS infants indicated of infection, with differentiated results between the full-term and premature groups, although both were very sensitive 
to changes in CRP. Premature infants showed a prominent decrease in white blood cells and a large proportion had neutropenia, while full-term infants showed an increase in

Table 1. Incidence and mortality of NNS in Shaanxi Province: 2008-2010.

\begin{tabular}{ccccc}
\hline Group & 2008 & 2009 & 2010 & Total \\
\hline Admission in neonatal department, $\mathrm{n}$ & 4972 & 5501 & 5501 & 16,642 \\
HAI, n & 139 & 113 & 105 & 357 \\
HAI/Admission, \% & 2.8 & 2.3 & 1.7 & 2.1 \\
NNS, n & 52 & 42 & 45 & 139 \\
NNS/HAI, \% & 37.4 & 37.1 & 42.8 & 38.9 \\
Incidence of NNS, \% & 1 & 0.76 & 0.72 & 0.83 \\
Death from NNS, n & 12 & 9 & 8 & 29 \\
Mortality of NNS, \% & 23 & 21.4 & 17.1 & 20.8 \\
\hline
\end{tabular}

HAI: Hospital Acquired Infection, NNS: Nosocomial Neonatal Sepsis.

Table 2. Clinical manifestation and disease burden of NNS in Shaanxi Province: 2008-2010.

\begin{tabular}{|c|c|c|c|}
\hline Group & Preterm & Full-term & P Value \\
\hline $\mathrm{N}, \%$ & $84(60.4)$ & & $55(39.6)$ \\
\hline Gestational age, weeks & $32 \pm 3.7$ & $38 \pm 1.9$ & 0.007 \\
\hline Birth weight, $g$ & $1765 \pm 789$ & $3246 \pm 881$ & 0.043 \\
\hline Gender (Male/Female) & $2.6: 1$ & $1.9: 1$ & 0.45 \\
\hline Average age of onset, days & 16.5 & 6.7 & 0.002 \\
\hline \multicolumn{4}{|l|}{ Clinical manifestation, $\mathrm{n}(\%)$} \\
\hline Apnea & $72(85.7)$ & $4(7.3)$ & 0 \\
\hline Poor response & $65(77.3)$ & $12(21.8)$ & 0 \\
\hline Poor feeding & $44(52.3)$ & $35(63.6)$ & 0.222 \\
\hline Gastric retention & $15(17.8)$ & $1(1.8)$ & - \\
\hline Hyperthermia & $6(7.1)$ & $11(20)$ & 0.033 \\
\hline Hypothermia & $21(25)$ & $5(9)$ & 0.025 \\
\hline Dyspnea stressed & $19(22.6)$ & $6(10.9)$ & 0.113 \\
\hline No weight increase & $69(82.1)$ & $41(74.5)$ & 0.294 \\
\hline Jaundice exacerbation & $15(17.8)$ & $32(58.1)$ & 0 \\
\hline Abdominal distention & $35(41.6)$ & $11(20)$ & 0.01 \\
\hline Abnormal defecation & $11(13.0)$ & $8(14.5)$ & 0.806 \\
\hline Mortality, n (\%) & $25(29.7)$ & $25(29.7)$ & 0.001 \\
\hline Death due to quit treatment, $\mathrm{n}(\%)$ & $6(24)$ & $1(25)$ & - \\
\hline Average hospitalization, days & 31 & 13 & 0.002 \\
\hline Average cost (RMB, Yuan) & 19,350 & 6,843 & 0.007 \\
\hline
\end{tabular}


Table 3. Laboratory examination of NNS in Shaanxi Province: 2008-2010.

\begin{tabular}{cccc}
\hline Group & Preterm & Full-term & P Value \\
\hline $\mathrm{N}, \%$ & $84(60.4)$ & $55(39.6)$ & - \\
$\mathrm{WBC}>20 \times 109 / \mathrm{L}$ & $36(42.8)$ & $38(69.0)$ & 0.003 \\
$\mathrm{WBC}<5 \times 109 / \mathrm{L}$ & $38(45.2)$ & $10(18.1)$ & 0.001 \\
Neutropenia & $19(22.6)$ & $4(7.3)$ & - \\
I/T of granulocyte $>0.16$ & $25(29.7)$ & $19(34.5)$ & 0.58 \\
CRP $>8$ mg/L & $75(89.2)$ & $46(83.6)$ & 0.439 \\
PLT $<100 \times 109 / \mathrm{L}$ & $43(51)$ & $15(27)$ & 0.064 \\
Anemia & $52(61.9)$ & $9(16.3)$ & 0 \\
Acidosis & $21(25)$ & $9(16.3)$ & 0.293 \\
Hyperglycemia & $23(27.3)$ & $11(20)$ & 0.42 \\
\hline
\end{tabular}

WBC: white blood cell, CRP: C reaction protein, I/T: immature per total, PLT: platelets.

white blood cells. There was no statistically significant difference in terms of decreased platelets, metabolic acidosis, and hyperglycemia between the two groups $(\mathrm{P}>0.05)$.

\subsection{Pathogens}

The predominant organism cultured was Gram-negative (67.6\%) followed by Grampositive (22.3\%), and Candida albicans (7.9\%). The most common Gram-negative organism identified in NNS was Klebsiella spp. (24.5\%), followed by E. coli (15.1\%) and E. cloacae (8.6\%). The most common Gram-positive organism detected in NNS was Coagulase-negative staphylococci (CONS) (8.6\%), followed by Staphylococcus aureus (7.9\%) and Enterococcus faecalis (3.7\%).

\subsection{Antibiotic Sensitivity}

The antibiotics resistant to Klebsiella pneumonia were ampicillin (91.3\%), piperacillin (62\%), and cefotaxime sodium (59.7\%). The sensitive antibiotics were meropenem (100\%), imipenem (100\%), and cefepime (93.5\%). The antibiotics resistant to E. coli were ampicillin (83.4\%), cefotaxime sodium (53.2\%), and cefuroxime sodium (47.5\%). The antibiotics resistant to E. cloacae were ampicillin (84.1\%), piperacillin (52.3\%), and ceftriaxone (44\%). The sensitive antibiotics of these two bacteria were meropenem (100\%), imipenem (100\%), and cefoperazone sulbactam (85.6\% and $83.2 \%)$. The antibiotics resistant to CONS were penicillin (82\%), erythromycin (74.1\%), and cotrimoxazole (52.5\%). The sensitive antibiotics of CONS were vancomycin (100\%), rifampicin (96\%), and minocycline (87.7\%). The antibiotics resistant to S. aureus were penicillin (68.3\%), erythromycin (65.2\%), and rifampicin (59\%). The antibiotics resistant to Enterococcus faecium were penicillin (86\%), oxacillin (57\%), and cotrimoxazole (42\%). The susceptible antibiotics of these two were vancomy (100\%), teicoplanin (100\%), cinlevofloxacin levofloxacin (76.5\%), and ciprofloxacin (72.3\%) (Table 4). 
Table 4. Pathogenic distribution of NNS in Shaanxi Province: 2008-2010.

\begin{tabular}{|c|c|c|c|}
\hline Pathogen & Preterm & Full-term & P Value \\
\hline $\mathrm{N}$ & 84 & 55 & 139 \\
\hline Gram-positive bacteria & $18(21.4)$ & $16(29.1)$ & $34(24.5)$ \\
\hline Coagulase-negative Staphylococci* & $6(7.0)$ & $6(11.0)$ & $12(8.6)$ \\
\hline MRCNS & $3(3.6)$ & $3(5.5)$ & $6(4.3)$ \\
\hline Staphylococcus aureus & $4(4.8)$ & $7(12.7)$ & $11(7.9)$ \\
\hline MRSA & $1(1.2)$ & $2(3.6)$ & $3(2.1)$ \\
\hline Enterococcus faecium & $4(4.8)$ & $1(1.8)$ & $5(3.7)$ \\
\hline Enterococcus faecalis & $2(2.4)$ & 0 & $2(1.4)$ \\
\hline Other Gram-positive bacteria & $2(2.4)$ & $2(3.6)$ & $4(2.9)$ \\
\hline Gram-negative bacteria & $57(67.9)$ & $37(67.3)$ & $94(67.6)$ \\
\hline Klebsiella pneumonia & $20(23.8)$ & $11(20)$ & $31(22.3)$ \\
\hline ESBL positive & $13(15.5)$ & $4(7.3)$ & $17(12.2)$ \\
\hline Klebsiella terrigena & $4(4.8)$ & $4(7.3)$ & $8(5.8)$ \\
\hline Escherichia coli & $14(16.6)$ & $7(12.7)$ & $21(15.1)$ \\
\hline Enterobacter cloacae & $7(8.3)$ & $5(9.2)$ & $12(8.6)$ \\
\hline Pseudomonas spp. & $4(4.8)$ & $2(3.6)$ & $6(4.3)$ \\
\hline Bauman acinetobacter spp. & $3(3.6)$ & $1(1.8)$ & $4(2.9)$ \\
\hline Serratia spp. & $3(3.6)$ & $2(3.6)$ & $5(3.6)$ \\
\hline Other enterobacteriaceae & $1(1.2)$ & $3(5.5)$ & $4(2.9)$ \\
\hline Other Gram-negative bacteria & $1(1.2)$ & $2(3.6)$ & $3(2.1)$ \\
\hline Fungi & $9(10.7)$ & $2(3.6)$ & $11(7.9)$ \\
\hline
\end{tabular}

MRCNS: Methicillin Resistant Coagulase Negative Staphylococcus, MRSA: Methicillin Resistant Staphylococcus Aureus, ESBL: Extended Spectrum Beta Lactamases.

\section{Conclusion}

The overall incidence of NNS was $0.83 \%$ of the total 16,642 admitted neonates in the 9 hospitals, and the mortality rate was $20.8 \%$ in Shaanxi Province from 2008 to 2010 . The pathogen was mainly Gram-negative (67.6\%), Gram-positive (24.5\%), or fungi (7.9\%). Antibiotics had varying degrees of resistance, and the preterm group and full-term group showed significant differences in initial clinical manifestations and laboratory examinations. The preterm infants with NNS had higher mortality and heavier disease burden, which leads to abandoning of the treatment.

\section{Discussion}

\subsection{Limitations of the Study}

This was a retrospective study based on admitted patients but not birth population be- 
cause of unavailability of an established nationwide medical information database in China. The positive rate of blood culture was not $100 \%$ [8], and if we take into account the cases of false-negative blood culture, the prevalence of NNS will be higher.

\subsection{Incidence and Mortality}

Huskins reported that rates of neonatal sepsis varied from 6.5 to 38 per 1000 live hospital-born babies. The bloodstream infection rates were 3 - 20 times higher in developing countries than the rates of 1 - 5 per 1000 live births reported in developed countries [9]. Stoll found that the incidence of LOS was 5.36-11.6\% in hospitalized neonates [10]. Yi reported that sepsis in NICU was $19.8 \%$ and mortality was $10 \%-50 \%$ in China [11].

Shaanxi is an underdeveloped province located in the northwestern China, with a population of 37 million. The birth rate was $8.88,11.5$ and 10.24 per one thousand people respectively, and the rate of institutional delivery was $97.1 \%, 98.8 \%$, and $99.4 \%$ in 2008, 2009, and 2010, respectively. The increasing of neonatal in-patients was because of higher birth rate and improving infant care. From 2008 to 2010, neonatal mortality rate (NMR) was 13.99, 9.1, and 8.94 per one thousand live birth respectively, NNS mortality was accounted for $2.4,1.63$, and 1.3 per one thousand admitted respectively, which showed the same declining trend but only by a small margin $(0.11 \%$ vs. $0.5 \%)$ [12].

Both incidence and mortality of NNS obtained in our study were higher than those in developed countries. The incidence of sepsis was $4.06 \%$ among all NICU admissions in Taiwan. Mortality in LOS was 7\% [13]. Isaacs reported an incidence of sepsis of 6.6 per 1000 live births in 7 Australian NICUs, of which 75\% were LOS and overall mortality was $10 \%$ [14]. In 1998, in a cohort of 54 UK neonatal units that registered 3963 admissions, there was $204(5 \%)$ cases of sepsis, of which $16(8 \%)$ died [15]. The relatively low NNS incidence may be related to Chinese neonatal saving boundaries, which begin from gestational age of 28 weeks but not 23 weeks in developed countries. In our study, only one case was under 28 weeks and only two were ELBW infants. VLBW was reported in $27.9 \%$ of $93 \mathrm{LBW}$ infants, who were the major contributors of NNS [16].

In our study, only $40 \%$ newborns had insurance (NCMS) support, which provided a reimbursement of $30 \%$ - 50\%. In Shaanxi Province, the average disposable per capita income was 12,858, 14,129, and 15,695 Yuan for city residents, and 4390, 4980, and 5375 Yuan for rural residents in 2008, 2009, and 2010, respectively (6.7 Yuan = $1 \$$ ). Compared with the income, the disease burden was heavy, especially for rural residents with premature infants [17]. High giving-up rates resulted from economic difficulties and the refractory nature of the disease, which strongly suggested that not only treatment level but also insurance coverage should be improved to reduce NNS mortality in this region.

\subsection{Clinical Manifestations, Laboratory Examinations, and Early Diagnosis}

Early diagnosis of neonatal sepsis is important, but clinical signs are neither specific nor 
uniform [18]. Many markers have been developed for early detection, including cellsurface markers (CD11b, CD64, CD69), chemokines and cytokines (IL-6, IL-8, IL-10), and acute phase reactants (CRP, procalcitonin). However, no single infection marker is sensitive and specific enough to convince a doctor to use or withdraw antibiotic therapy in a sick infant. Developing diagnostic tools such as DNA arrays may be useful in the future [19].

Blood culture is the gold standard for the diagnosis of septicemia. However, it is not error-free because of the possibility of falsely sterile or insufficient sample, low-density bacteremia, or suppression of bacterial growth by earlier antibiotic administration [20]. Positive cultures reportedly ranged from $8 \%$ to $73 \%$ in the diagnosis of potential neonatal sepsis. The positive predictive value of blood routine test series was higher than the negative predictive value [21]. In underdeveloped regions, medical equipment and personnel are relatively insufficient for infants, and early recognition of atypical symptom experience is very important. We believe that it is more helpful to train medical staff and at the same time combine sepsis screening than to use biological markers or only monitoring.

\subsection{Pathogens and Antibiotic Sensitivity}

Jordan reported that $70 \%$ pathogens of LOS was Gram-positive bacteria, and fungi accounted for 15\% [22]. NICHD Neonatal Research Network showed that Gram-positive organisms were most commonly associated with LOS among VLBW infants [17]. In UK, neonatal Network demonstrated that Gram-positive organisms comprised 70\% of infections, Gram-negative organisms, 25\%, and fungi, 5\%, from 2006 to 2008. Of the Gram-positive pathogens, the top three were CONS (42\%), S. aureus (10\%), and S. enterococci (9\%). Of the Gram-negative pathogens, E. coli contributed 8\%, and Klebsiella spp. and Enterobacter spp., 5\% each, and Candida spp. was responsible for all of the fungal infections [23]. In Japan, 58 NNS cases were diagnosed in 871 NICU-admitted neonates, $44.8 \%$ of which resulted from Staphylococcus species, including MRSA (25.9\%), MSSA (3.4\%), and CNS (15.5\%) [24]. Kamath found that Gram-negative contributed to $71.8 \%$ of NNS, and the predominant pathogens were extended spectrum beta lactamase (ESBL)-producing Klebsiella species and MRSA [25]. In China, increasing Gram-negative bacilli dominance was reported, most of which was K. pneumoniae and E. coli. Nearly $6 \%$ of E. coli strains were reported as resistant to most cephalosporins and $83 \%$ of S. aureus strains were reported as resistant to oxacillin [26]. The differences in pathogens indicated that we cannot choose antibiotics based on the experience in developed countries. For example, doctors in developed countries use ampicillin and gentamicin as primary antibiotics, but it is not feasible in China because most pathogens of NNS are resistant to ampicillin; further, gentamicin is not permitted to be used for newborns in Chinese routine guidelines. The choice of antibiotics for an infant with suspected sepsis depends upon the predominant pathogens and antibiotic sensitivity pattern of a given region. However, routine use of these "high-level" antibiotics might increase the risk of infections with ESBL-positive organisms. We have also found 
that doctors use enzyme penicillin or cephalosporin as prophylactic antibiotics in our study, which may increase resistance to bacteria [27], for there is a challenge in management of extensive use of broad-spectrum antibiotics and long-term empirical use of antibiotics.

\subsection{Therapy and Prevention}

NNS needs comprehensive management, and the most effective method is hygiene of the hand. However, there are two primary factors that contribute to poor hygiene of the hand in underdeveloped regions: shortage of nursing staff and lack of investment to control hospital-induced infection [28]. Studies have shown that breastfeeding can reduce the incidence of LOS, but exclusive breast feeding rate is only $1.4 \%$ in our study compared with $50 \%-90 \%$ in developed NICUs [29]. As indicated by our results, the infection control team of PICC catheter is insufficient. The success of the "One is Not Zero" philosophy requires strategies to prevent NNS, including improved hand hygiene, early breast milk feeding, limited use of invasive devices, rational use of antibiotics, and improved teamwork to contain infection [30].

\section{Acknowledgements}

We thank the investigators and institutions participated in the study of Shaanxi Collaborative Study Group for Nosocomial Neonatal Sepsis. This study was supported by Shaanxi Bureau of Health (No. 2010D017). We are grateful to Dr. Sun Bo from Children's Hospital of Fudan University, Shanghai, for his advice.

\section{Conflict of Interest}

The authors declare that they have no conflict of interest to disclose.

\section{References}

[1] Rajaratnam, J.K., Marcus, J.R., Flaxman, A.D., Wang, H., Levin-Rector, A., Dwyer, L., et al. (2010) Neonatal, Postneonatal, Childhood, and Under-5 Mortality for 187 Countries, 1970-2010: A Systematic Analysis of Progress towards Millennium Development Goal 4. Lancet, 375, 1988-2008. http://dx.doi.org/10.1016/S0140-6736(10)60703-9

[2] Lawn, J.E., Kerber, K., Enweronu-Laryea, C., et al. (2009) Newborn Survival in Low Resource Settings-Are We Delivering? BJOG, 116, 49-59. http://dx.doi.org/10.1111/j.1471-0528.2009.02328.x

[3] Rudan, I., Chan, K.Y., Zhang, J.S., et al. and Other Members of the WHO/UNICEF's Child Health Epidemiology Reference Group (CHERG) (2010) Causes of Deaths in Children Younger than 5 Years in China in 2008. Lancet, 375, 1083-1089. http://dx.doi.org/10.1016/S0140-6736(10)60060-8

[4] Liang, J., Zhen, X., Zhu, J., Mu, D., Li, X., Li, Y., et al. (2012) Neonatal Infection-Associated Mortality in China, a Population-Based Study, 2003-2008. Journal of Maternal-Fetal and Neonatal Medicine, 25, 2750-2755. http://dx.doi.org/10.3109/14767058.2012.664196

[5] Makhoul, I.R., Yacoub, A., Smolkin, T., et al. (2006) Values of C-Reactive Protein, Procalcitonin, and Staphylococcus-Specific PCR in Neonatal Late-Onset Sepsis. Acta Paediatrica, 95, 1218-1223. http://dx.doi.org/10.1080/08035250600554250 
[6] Stoll, B.J. (2001) Epidemiology of Early and Late-Onset Neonatal Infections in Kliegman. Saunders Elsevier: Philadelphia, PA: Nelson Textbook of Pediatrics, Richard E. Behrman.16th Edition.

[7] Chinese Ministry of Health (2001) Diagnostic Criteria for Nosocomial Infections. National Medical Journal of China, 81(5).

[8] Favre, B., Hugonnet, S., Correa, L., et al. (2005) Nosocomial Bacteremia: Clinical Significance of a Single Blood Culture Positive for Coagulase-Negative Staphylococci. Infect Control Hospital Epidemiology, 26, 697-702. http://dx.doi.org/10.1086/502605

[9] Zaidi, A.K., Huskins, W.C., Thaver, D., et al. (2005) Hospital-Acquired Neonatal Infections in Developing Country. Lancet, 365, 1175-1188. http://dx.doi.org/10.1016/S0140-6736(05)71881-X

[10] Stoll, B.J., Hansen, N., Fanaroff, A.A., et al. (2002) Late-Onset Sepsis in Very-Low Birth Weight Neonates: The Experience of the NICHD Neonatal Research Network. Pediatrics, 110, 285-291.

[11] Yi, J. (2010) Advances in Diagnosis and Treatment of Neonatal Sepsis. Chinese Journal of Neonatology, 25, 69-72.

[12] Shaanxi Province Health Department (2013) 2012 Maternal and Child Health Report of Shaanxi Province.

[13] Wu, J.H., Chen, C.Y., Tsao, P.N., et al. (2009) Neonatal Sepsis: A 6-Year Analysis in a Neonatal Care Unit in Taiwan. Pediatrics \& Neonatology, 50, 88-95. http://dx.doi.org/10.1016/S1875-9572(09)60042-5

[14] Isaacs, D., Barfield, C.P., Grimwood, K., et al. (1995) Systemic Bacterial and Fungal Infections in Infants in Australian Neonatal Units. Australian Study Group for Neonatal Infections. The Medical Journal of Australia, 162, 198-201.

[15] Stoll, B.J., Holman, R.C. and Schuchat, A. (1998) Decline in Sepsis-Associated Neonatal and Infant Deaths in the United States, 1979 through 1994. Pediatrics, 102, e18. http://dx.doi.org/10.1542/peds.102.2.e18

[16] Stoll, B.J., Gordon, T. and Korones, S.B. (1996) Late-Onset Sepsis in Very Low Birth Weight Neonates: A Report from the National Institute of Child Health and Human Development Neonatal Research Network. The Journal of Pediatrics, 129, 63-71. http://dx.doi.org/10.1016/S0022-3476(96)70191-9

[17] The Government of Shaanxi Province (2011) 2010 Shaanxi Provincial Government Work Report.

[18] Hornik, C.P., Fort, P., Clark, R.H., et al. (2012) Early and Late Onset Sepsis in Very-Low-Birth-Weight Infants from a Large Group of Neonatal Intensive Care Units. Early Human Development, 88, S69-S74. http://dx.doi.org/10.1016/S0378-3782(12)70019-1

[19] Srinivasan, L. and Harris, M.C. (2012) New Technologies for the Rapid Diagnosis of Neonatal Sepsis. Current Opinion in Pediatrics, 24, 165-171. http://dx.doi.org/10.1097/mop.0b013e3283504df3

[20] Jordan, J.A. and Durso, M.B. (2000) Comparison of 16S rRNA gene PCR and BACTEC9240 for Detection of Neonatal Bacteremia. Journal of Clinical Microbiology, 38, 2574-2578.

[21] Pammi, M., Flores, A., Lee Flang, M. and Versalovic, J. (2011) Molecular Assays in the Diagnosis of Neonatal Sepsis: A Systematic Review and Meta-Analysis. Pediatrics, 128, e973e985. http://dx.doi.org/10.1542/peds.2011-1208

[22] Lim, W.H., Lien, R., Huang, Y.C., et al. (2012) Prevalence and Pathogen Distribution of Neonatal Sepsis among Very Low Birth Weight Infants. Pediatrics \& Neonatology, 53, 228 234. http://dx.doi.org/10.1016/j.pedneo.2012.06.003 
[23] Vergnano, S., Menson, E., Kennea, N., et al. (2011) Neonatal Infections in England: The NeonIN Surveillance Network. Archives of Disease in Childhood-Fetal and Neonatal Edition, 96, F9-F14. http://dx.doi.org/10.1136/adc.2009.178798

[24] Babazono, A., Kitajima, H., Nishimaki, S., et al. (2008) Risk Factors for Nosocomial Infection in the Neonatal Intensive Care Unit by the Japanese Nosocomial Infection Surveillance (JANIS). Acta Medica Okayama, 62, 261-268.

[25] Kamath, S., Mallaya, S. and Shenoy, S. (2010) Nosocomial Infections in Neonatal Intensive Care Units: Profile, Risk Factor Assessment and Antibiogram. Indian Journal of Pediatrics, 77, 37-39. http://dx.doi.org/10.1007/s12098-010-0005-5

[26] Wu, J., Wu, B.Q. and Huang, J.J. (2012) Risk Factors and Pathogen Distribution in Premature Infants with Nosocomial Sepsis. Chinese Journal of Contemporary Pediatric, 14, 93-96.

[27] Adams-Chapman, I. and Stoll, B.J. (2002) Prevention of Nosocomial Infections in the Neonatal Intensive Care Unit. Current Opinion in Pediatrics, 14, 157-164.

http://dx.doi.org/10.1097/00008480-200204000-00003

[28] Zaidi, A.K., Huskins, W.C., Thaver, D., et al. (2005) Hospital-Acquired Neonatal Infections in Developing Countries. The Lancet, 365, 1175-1188. http://dx.doi.org/10.1016/S0140-6736(05)71881-X

[29] Schanler, R.J. (2011) Outcomes of Human Milk-Fed Premature Infants. Seminars in Perinatology, 35, 29-33. http://dx.doi.org/10.1053/j.semperi.2010.10.005

[30] Shane, A.L. and Stoll, B.J. (2013) Recent Developments and Current Issues in the Epidemiology, Diagnosis, and Management of Bacterial and Fungal Neonatal Sepsis. American Journal of Perinatology, 30, 131-141. http://dx.doi.org/10.1055/s-0032-1333413

\section{Submit or recommend next manuscript to SCIRP and we will provide best service for you:}

Accepting pre-submission inquiries through Email, Facebook, LinkedIn, Twitter, etc. A wide selection of journals (inclusive of 9 subjects, more than 200 journals)

Providing 24-hour high-quality service

User-friendly online submission system

Fair and swift peer-review system

Efficient typesetting and proofreading procedure

Display of the result of downloads and visits, as well as the number of cited articles

Maximum dissemination of your research work

Submit your manuscript at: http://papersubmission.scirp.org/

Or contact ojped@scirp.org 